\title{
Preventing harmful effects of epileptic seizures on cerebrovascular functions in newborn pigs: does sex matter?
}

\author{
Jianxiong Liu ${ }^{1}$, Massroor Pourcyrous ${ }^{1}$, Alex L. Fedinec ${ }^{1}$, Charles W. Leffler ${ }^{1}$ and Helena Parfenova ${ }^{1}$
}

BACKGROUND: The potential contribution of sex-related variables to cerebrovascular functions in neonates remains elusive. Newborn piglets provide a translationally relevant model for studying the effects of seizures in the neonatal brain. The present study investigated whether sex differences contribute to cerebrovascular functions in healthy and epileptic newborn pigs.

METHODS: Epileptic seizures were induced in female and male newborn pigs by bicuculline. An antioxidant drug, the carbon monoxide-releasing molecule CORM-A1, was administered enterally before or during seizures. The responses of pial arterioles to endothelium-, astrocyte-, and vascular smooth muscle-dependent vasodilators were tested in intact and 48-h postictal piglets using the cranial window technique.

RESULTS: In intact newborn pigs, we did not observe any sex-related differences in cerebrovascular functions. In the postictal male and female newborn pigs, a marked reduction in responses of pial arterioles to endothelium- and astrocytedependent vasodilators was detected. CORM-A1, administered before or during seizures, greatly improved the outcome of seizures on cerebrovascular functions in both male and female piglets.

CONCLUSION: We found no evidence of sex-related differences in cerebral vasodilator functions in control and epileptic newborn pigs. In both male and female newborns, epileptic seizures lead to prolonged cerebral vascular dysfunction that is effectively prevented by CORM-A1 therapy.

S eizures are the most common neurological event in human newborns that frequently lead to neuronal damage and adverse neurological outcome. Epileptic seizures in the developing brain produce lifelong developmental, cognitive, and motor deficits (1-4). Seizures have harmful long-lasting effects on cerebral blood flow (CBF) regulation. Cerebral vascular health is an important contributing factor to brain homeostasis and neuronal health. Newborn piglets provide a well-established and translationally relevant model for studying the cerebrovascular effects of epileptic seizures in the neonatal brain. In newborn pigs of both sexes, epileptic seizures lead to cerebral vascular injury, apoptosis, and long- term insufficiencies in cerebral vascular functions, including sustained loss of endothelium- and astrocyte-mediated regulation of CBF (5-8). Clinical studies in newborn babies with epileptic seizures caused by neonatal hypoxic-ischemic encephalopathy and severe intraventricular hemorrhage (III-IV) revealed the occurrence of cerebral vascular damage diagnosed by the surge of circulating endothelial cells of brain origin in peripheral blood (9). Cerebral vascular insufficiencies have been also detected in epileptic patients $(10,11)$.

Brain oxidative stress caused by seizures is the major contributor to cerebral vascular injury and CBF dysregulation. Adverse cerebrovascular effects of neonatal seizures can be prevented by treatment with systemic antioxidants (6). The antioxidant compound CORM-A1, a carbon monoxide (CO) donor, is among the most effective cerebroprotective drugs used to reduce adverse cerebrovascular effects of seizures in the neonatal brain (6-8).

There is a growing interest in the potential contribution of sex-related variables to cerebrovascular functions in healthy and sick neonates. The foundation for potential sexual dimorphism in the neonatal mammalian brain is provided by the fact that male and female sex steroids are produced in the neonatal brain (cortex, hypothalamus, and hippocampus) at high levels independently of the circulating steroids during the first days of the postnatal period (12). Relatively high circulating levels of estradiol and testosterone are also detectable in human infants shortly after birth, reaching a transient maximum by 1-2 months ("transient minipuberty" in early infancy) (13). Furthermore, hormone-independent components of sex-biased gene expression may contribute to sex-dependent differences in physiological functions (13-15). Animal studies suggest that male infants have a higher propensity to brain injury caused by inflammation, hypoxiaischemia, stroke, and trauma (16-23).

Clinical findings in neonates have produced controversial findings on sexual dimorphism in neonatal brain disease and the efficiency of neuroprotective therapy. In preterm low-birthweight infants, overall better survival and neurodevelopmental outcomes in female infants have been reported (24). However, large-scale clinical studies in newborns with hypoxic-ischemic encephalopathy did not reveal any sex-related differences in the outcomes of hypothermia therapy on reducing mortality and 
neurodevelopmental disabilities in survivors $(25,26)$. Clearly, controlled studies in various translationally relevant animal models of neonatal brain disease are required to identify sex-specific differences in the outcome and the potential need for personalized therapies applicable to specific pathological conditions $(21,26)$.

The contribution of sex differences to the incidence and outcome of seizures in newborns remains controversial. Reports from animal studies suggest that sex-specific differences contribute to the occurrence of early life seizures, with male newborns being more prone to seizures (27-29). Several reports on human neonates indicate that sex-related differences appear to involve a slightly higher incidence of seizures in male newborns (26,30). However, large-scale clinical studies do not provide a strong case for the contribution of sex to the incidence and outcome of seizures (27,31-34).

The question of whether there are sex-related differences in the cerebral vascular outcome of seizures remains unanswered. Our study on newborn piglets was designed to gain an understanding of potential sex-related mechanisms that may contribute to cerebrovascular disease caused by epileptic seizures and to address the need for developing personalized neuroprotective therapies for newborns. We tested the hypothesis that in male newborn piglets, seizures produce a greater cerebral vascular dysfunction that is largely resistant to antioxidant therapy. Using randomly selected male and female newborn pigs, we evaluated a variety of endotheliumand/or astrocyte-dependent and -independent components of $\mathrm{CBF}$ regulation in healthy and epileptic newborn pigs. We also investigated the effectiveness of antioxidant therapy with CORM-A1 in improving the cerebral vascular outcome of seizures in male and female newborn pigs.

\section{METHODS}

Newborn piglets (1-5 days old, $1.5-2.5 \mathrm{~kg}$, either sex) were purchased from a commercial breeder. The distribution of male and female piglets in the experimental groups was equal. Veterinary care was provided by the Department of Comparative Medicine. All experimental protocols were approved by the Animal Care and Use Committee of the University of Tennessee Health Science Center in accordance with the National Institutes of Health guidelines for the care and use of animals in research and followed the ARRIVE guidelines.

\section{Bicuculline Model of Neonatal Seizures}

Epileptic seizures were induced by bicuculline, a $\mathrm{GABA}_{\mathrm{A}}$ receptor blocker that enhances excitatory neurotransmission. The piglets were anesthetized with ketamine/xylazine $(33 / 2 \mathrm{mg} / \mathrm{kg}$, intramuscular) and intubated with endotracheal tubes through the mouth and were ventilated with $4 \% \mathrm{CO}_{2}, 21 \% \mathrm{O}_{2}, 75 \% \mathrm{~N}_{2}$ to maintain physiological levels of blood gases $(5,8,35)$. The body temperature was maintained at $37-38^{\circ} \mathrm{C}$ by a servo-controlled heating pad. Pancuronium (Astra Pharmaceutical Products; Westborough, MA; $0.2 \mathrm{mg} / \mathrm{kg}$ ) was administered via the ear vein before inducing seizures to block twitching. Bicuculline (R\&D Systems, Minneapolis, MN; $3 \mathrm{mg} / \mathrm{kg}$, intraperitoneal) induces generalized tonic-clonic seizures that last for $\sim 2 \mathrm{~h}$ (35). The heart rate, a reliable indicator of seizure activity, was continuously monitored. CORM-A1 (Dalton Pharma Services; Toronto, Canada; $2 \mathrm{mg} / \mathrm{kg}$ ) was administered to anesthetized pigs enterally via a gastric feeding tube as we described previously (8).
The animals were kept on the ventilator for $2-3 \mathrm{~h}$ after bicuculline administration until the seizure activity subsides, and pancuronium/ anesthesia wears off. After fully regaining consciousness, the piglets were transferred to the animal care facility and kept in warmed cages with food and drink ad libitum for 2 days. The recovery after seizures is largely uneventful. During the delayed postictal period, we did not observe behavioral difficulties that include breathing, standing, walking, or lack of interest in food. Postictal cerebral vascular functions were tested 2 days after seizures.

\section{Experimental Groups}

The experimental protocols were designed to investigate whether sex-related differences contribute to cerebral vascular functions in healthy and epileptic newborn pigs. The male and female piglets ( $n=4-5$ animals in each group) were selected randomly and divided into the following groups: (1) intact control Group I (no seizures); (2) saline seizures Group II (10 ml saline, $10 \mathrm{~min}$ before bicuculline); (3) preventive CORM-A1 seizure Group III ( $2 \mathrm{mg} / \mathrm{kg}$ in $10 \mathrm{ml}$ saline, $10 \mathrm{~min}$ before bicuculline); and (4) therapeutic CORM-A1 Group IV ( $2 \mathrm{mg} / \mathrm{kg}$ in $10 \mathrm{ml}$ saline, $20 \mathrm{~min}$ after bicuculline).

Intravital Microscopy for Evaluating Cerebral Vascular Functions Cerebral vascular functions were tested using intravital microscopy via closed cranial windows that allows direct observation of pial arterioles. Intact control and 48 -h postictal piglets were anesthetized with ketamine/xylazine $(33 / 2 \mathrm{mg} / \mathrm{kg}$, intramuscular) and maintained with $\alpha$-chloralose $(50 \mathrm{mg} / \mathrm{kg}$, intravenous) $(5,8)$. Core body temperature was kept at $37-38^{\circ} \mathrm{C}$ using a servo-controlled heating pad. Piglets were intubated by tracheostomy and ventilated with room air using an infant mechanical ventilator. Femoral arterial and venous catheters were inserted for the monitoring of cardiovascular parameters and blood gases. The blood gases were maintained at a normal physiological range $\left(\mathrm{pH} \approx 7.4 ; \quad \mathrm{PaCO}_{2} \approx 40 \mathrm{~mm} \mathrm{Hg}\right.$; $\mathrm{PaO}_{2} \approx 80 \mathrm{~mm} \mathrm{Hg}$ ). Closed cranial windows were placed over the left parietal cortex area via the craniotomy as we have described in detail before $(5,8)$. Arteriolar diameters were measured using a digital videomicrometer connected to a Wild Heerbrugg M3B Type-S intravital microscope (Leica Microsystems, Buffalo Grove, IL, USA). Four to six pial arterioles $(40-80 \mu \mathrm{m}$ diameter) were selected for observation in each piglet.

Cerebral vascular functions essential for CBF regulation were tested by responses of pial arterioles to endothelium- and astrocytedependent, and to vascular smooth muscle-dependent vasodilator stimuli. The vasodilators were applied directly to the brain surface under the cranial window. We used: (i) the endothelium-dependent vasodilator bradykinin $\left(10^{-6} \mathrm{M}\right)$, (ii) the endothelium- and astrocyte-dependent vasodilators glutamate $\left(10^{-4} \mathrm{M}\right)$, quisqualate $\left(10^{-4} \mathrm{M}\right)$, and heme $\left(10^{-5} \mathrm{M}\right)$, (iii) the astrocyte-dependent vasodilator adenosine diphosphate (ADP, $10^{-4} \mathrm{M}$ ), and (iv) the vascular smooth muscle-dependent vasodilator sodium nitroprusside $\left(10^{-5} \mathrm{M}\right)$ that acts independently of endothelial or astrocytic influences. All vasodilators were from Sigma (St Louis, MO).

\section{Statistical Analysis}

Values are presented as mean $\pm \mathrm{SE}$ of the absolute values or as percentage changes from control value. Analysis from prior studies shows that a sample size of 5 will yield statistical significance with power of 0.8 . To evaluate the effects of seizures on cerebral vascular function in female and male newborn pigs, data were analyzed by Student's $t$-test and analysis of variance. Differences were considered statistically significant for $P<0.05$.

\section{RESULTS}

Systemic Circulatory Parameters in Male and Female Newborn Pigs We assessed the systemic circulatory parameters in female and male newborn pigs in all four experimental groups (Table 1, Figure 1). In the intact control group (Group I), no 
Table 1. Systemic parameters in female $(F)$ and male $(M)$ newborn piglets

\begin{tabular}{|c|c|c|c|c|c|c|}
\hline Group & MABP $(\mathrm{mm} \mathrm{Hg})$ & HR (bpm) & $\mathrm{PaCO}_{2}(\mathrm{~mm} \mathrm{Hg})$ & $\mathrm{PaO}_{2}(\mathrm{~mm} \mathrm{Hg})$ & $\mathrm{pH}$ & $T\left({ }^{\circ} \mathrm{C}\right)$ \\
\hline $\mathrm{F}-\mathrm{I}$ & $59 \pm 2$ & $160 \pm 9$ & $36 \pm 1$ & $89 \pm 3$ & $7.36 \pm 0.01$ & $37.4 \pm 0.1$ \\
\hline$M-1$ & $61 \pm 2$ & $170 \pm 10$ & $36 \pm 1$ & $87 \pm 2$ & $7.35 \pm 0.01$ & $37.3 \pm 0.1$ \\
\hline$M-I I$ & $70 \pm 2$ & $150 \pm 7$ & $38 \pm 1$ & $80 \pm 1$ & $7.36 \pm 0.01$ & $37.5 \pm 0.1$ \\
\hline$F-I I I$ & $66 \pm 1$ & $150 \pm 8$ & $34 \pm 1$ & $92 \pm 3$ & $7.40 \pm 0.01$ & $37.5 \pm 0.1$ \\
\hline$M-I V$ & $58 \pm 2$ & $140 \pm 5$ & $31 \pm 2$ & $94 \pm 2$ & $7.34 \pm 0.01$ & $37.4 \pm 0.1$ \\
\hline
\end{tabular}

$H R$, heart rate; MABP, mean arterial blood pressure.

Experimental groups: Group I: intact control group (no seizures); Group II: saline seizures group (48 h after seizures); Group III: preventive CORM-A1 seizure group (48 h after seizures); Group IV: therapeutic CORM-A1 group (48 h after seizures). $N=5$ piglets in each group.

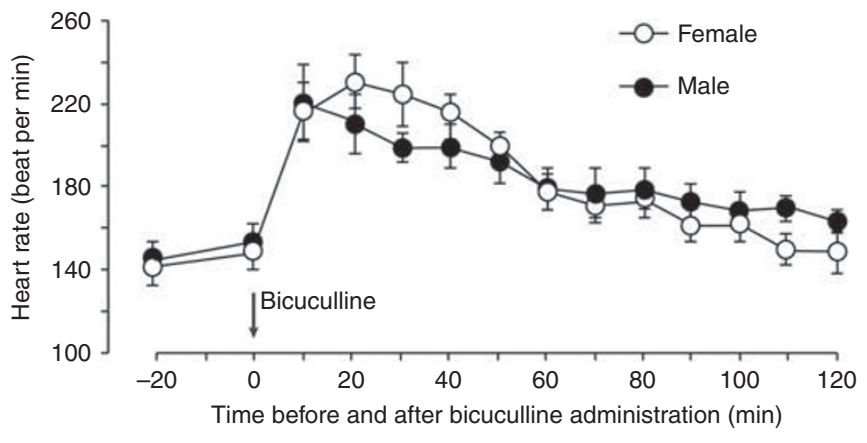

Figure 1. The dynamics of the ictal tachycardia response to seizures in female and male newborn pigs (Group II). $N=5$ piglets in each group.

differences between female and male pigs were observed in arterial blood pressure, heart rate, arterial blood gases and $\mathrm{pH}$, or body temperature. The tachycardia response to bicucullineinduced seizures in female and male piglets (Group II) was identical in amplitude, dynamics, and duration (Figure 1). No sex-related differences in the systemic parameters were observed in 48-h postictal female and male pigs, either salineor CORM-A1-treated ones (Groups II, III, and IV). Overall, we found no evidence of sex differences in arterial blood pressure and heart rate in control, ictal, and postictal newborn pigs.

\section{Cerebral Vascular Functions in Control Male and Female Newborn Pigs (Group I)}

Cerebral vascular function was evaluated by responses of pial arterioles to a variety of vasoactive stimuli that produce vasodilation by acting on vascular smooth muscle directly (sodium nitroprusside, SNP) or via endothelium- and/or astrocyte-mediated influences (hypercapnia, bradykinin, glutamate, quisqualate, ADP, heme). First, we asked the question whether there are sex-related differences in distinct cerebral vascular functions in healthy control newborns (Group I, Figure 2). The responses of pial arterioles to the nitric oxide (NO) donor SNP $\left(10^{-6} \mathrm{M}\right)$ that produces smooth muscle relaxation independently of endothelial or astrocytic influences were identical in control female and male piglets.

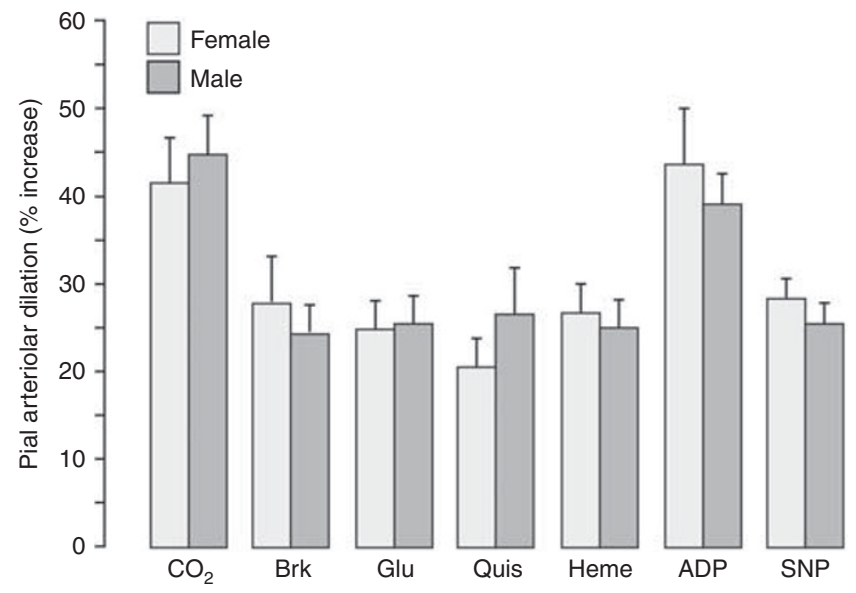

Figure 2. Absence of sex-related differences in endothelium-, astrocyte, and smooth muscle-dependent components of the CBF regulation in intact newborn pigs. Cerebral vascular functions in female and male newborn pigs (intact control Group I) were tested by dilator responses of pial arterioles to the endothelium-dependent vasodilators hypercapnia $\left(\mathrm{PaCO}_{2}, 70-80 \mathrm{~mm} \mathrm{Hg}\right)$, bradykinin $\left(\mathrm{BK}, 10^{-6} \mathrm{M}\right)$, endothelium/astrocyte-dependent dilators glutamate ( $\left.\mathrm{Glu}, 10^{-4} \mathrm{M}\right)$, quisqualic acid (Quis, $10^{-4} \mathrm{M}$ ), and heme $\left(10^{-5} \mathrm{M}\right)$, to astrocytedependent dilator ADP $\left(10^{-4} \mathrm{M}\right)$, and to vascular smooth muscledependent dilator sodium nitroprusside (SNP, $\left.10^{-6} \mathrm{M}\right) . N=5$ piglets in each group.

Endothelium-dependent vasodilation responses of pial arterioles to hypercapnia $\left(\mathrm{PaCO}_{2}, \quad 70-80 \mathrm{~mm} \mathrm{Hg} ; \mathrm{PaO}_{2}\right.$, $\sim 90 \mathrm{~mm} \mathrm{Hg} ; \mathrm{pH} \sim 7.0)$ and topical bradykinin $\left(10^{-6} \mathrm{M}\right)$ were identical in control female and male newborn pigs. We found no differences between female and male piglets in vasodilator responses to astrocyte-mediated vasodilator $\operatorname{ADP}\left(10^{-4} \mathrm{M}\right)$. Pial arteriolar responses to topical glutamatergic vasodilators that involve both endothelium- and astrocyte-mediated influences-i.e., glutamate $\left(10^{-4} \mathrm{M}\right)$ and AMPA/Kainate receptor agonist quisqualate $\left(10^{-4} \mathrm{M}\right)$-were identical in female and male newborn pigs. Similarly, we found no differences in responses of pial arterioles to heme $\left(10^{-5} \mathrm{M}\right)$, which initiates vasodilation by increasing endogenous production of vasodilator $\mathrm{CO}$ by both endothelial and astrocytic components of the neurovascular unit. Overall, in the intact 
control group of newborn pigs, we found no sex differences in cerebral vascular functions as tested by the responses of pial arterioles to a variety of endothelium-, astrocyte-, and vascular smooth muscle-dependent vasodilator stimuli.

\section{Deleterious Effects of Seizures on Cerebral Vascular Functions in Male and Female Newborn Pigs (Group II)}

To assess potential sex differences in deteriorating effects of seizures on cerebral vascular functions, we evaluated cerebral vascular functions in female and male newborn pigs during the 48 -h postictal period (Figure 3 ). In both female and male postictal piglets, cerebral vascular responses to SNP were not reduced compared to those of the intact controls, suggesting that vascular smooth muscle function was not adversely affected in either sex by seizures (Figure 3). In contrast, a significant reduction in cerebral vascular responses to endothelium- and/or astrocyte-dependent vasodilators was observed in female and male postictal piglets (Group II). Forty-eight hours after seizures, the vasodilator responses to bradykinin, glutamate, quisqualate, $\mathrm{ADP}$, and heme were reduced by $40-70 \%$ as compared with the responses in intact control pigs (Figure 3 ). These data strongly suggest that neonatal seizures produce sustained loss of endothelium- and astrocyte-mediated cerebral vascular functions in both male and female piglets. Remarkably, neither male nor female newborn pigs were found to be more susceptible to seizureinduced cerebral vascular dysfunction.

\section{CORM-A1 Therapy Prevents Postictal Cerebral Vascular}

Dysfunction in Female and Male Newborn Pigs (Groups III and IV) CORM-A1 administered via enteral or parenteral routes provides the delivery of cytoprotective CO to the brain (33). We addressed the question of whether sex-related differences contribute to the cerebroprotective potency of CORM-A1 against seizure-induced cerebral vascular dysfunction. Female and male newborn pigs were treated with CORM-A1 (2 mg/ $\mathrm{kg}$ ) shortly before seizures (Group III) or during the advanced ictal period (Group IV). Postictal cerebral vascular function was investigated $48 \mathrm{~h}$ after seizures. In both female and male piglets, CORM-A1, enterally administered either before seizures or during the advanced ictal period, effectively prevented the postictal loss of cerebral vascular responses to bradykinin, glutamate, and quisqualate (Figure 4), and to ADP and heme (Figure 5). The responses of pial arterioles to SNP during the $48 \mathrm{~h}$ postictal period remained at the intact control levels in either saline- or CORM-A1-treated postictal groups (Figure 5). No sex-related differences in the vasodilator responses to SNP have been detected in the control or any of the postictal group.

\section{DISCUSSION}

We investigated whether sex-related differences contribute to cerebrovascular functions in healthy and epileptic newborn pigs. Our experimental results did not support our tested hypothesis that in male newborn pigs, seizures produce a greater cerebral vascular dysfunction that is resistant to

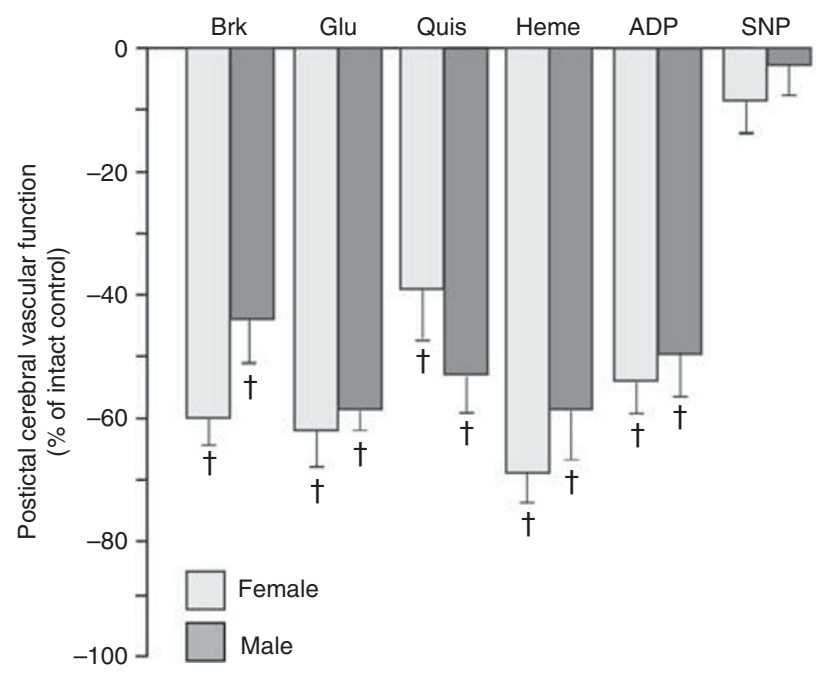

Figure 3. Neonatal seizures produce long-term adverse outcome on endothelium- and/or astrocyte-dependent components of the CBF regulation in both female and male newborn pigs (Group II). Cerebral vascular responses to the endothelium-dependent vasodilator bradykinin (BK, $10^{-6} \mathrm{M}$ ), endothelium/astrocyte-dependent dilators glutamate (Glu, $10^{-4} \mathrm{M}$ ), quisqualic acid (Quis, $10^{-4} \mathrm{M}$ ), and heme $\left(10^{-5} \mathrm{M}\right)$, to astrocyte-dependent dilator ADP $\left(10^{-4} \mathrm{M}\right)$, and to vascular smooth muscle-dependent dilator sodium nitroprusside (SNP, $10^{-6} \mathrm{M}$ ) were examined in female and male newborn pigs $48 \mathrm{~h}$ after the seizure. Postictal cerebral vascular function in saline seizures group (Group II) is expressed as the \% reduction of the intact control group responses. $N=5$ piglets in each group. ${ }^{\dagger} P<0.05$ compared with the intact control (Group I).

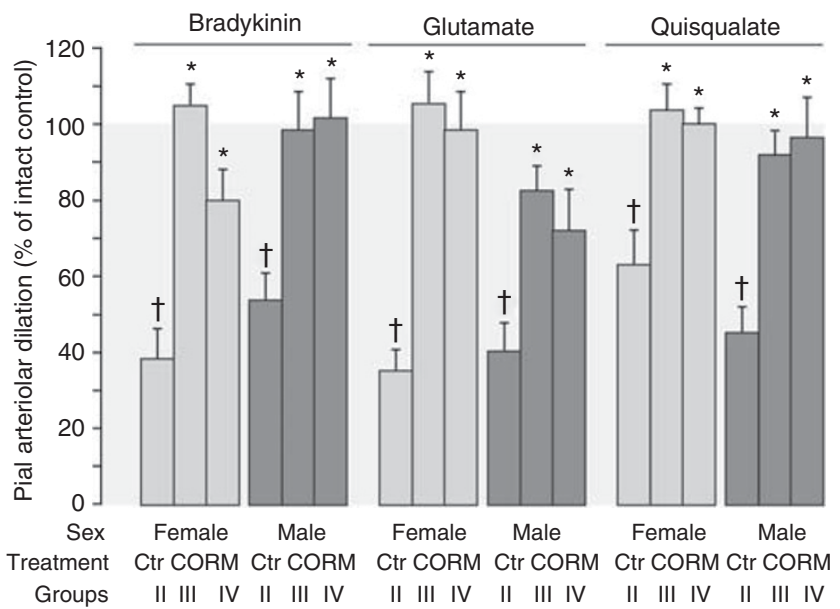

Figure 4. CORM-A1 therapy prevents postictal loss of cerebral vascular responses to bradykinin and glutamatergic vasodilators in female and male newborn pigs (Groups III and IV). CORM-A1 (2 mg/kg) was administered 10 min before (Group III) or 20 min after bicuculline (Group IV). Cerebral vascular responses to topical bradykinin $\left(10^{-6} \mathrm{M}\right)$, glutamate $\left(10^{-4} \mathrm{M}\right)$, and quisqualic acid $\left(10^{-4} \mathrm{M}\right)$ were examined $48 \mathrm{~h}$ after seizures in piglets, either untreated (Group II) or treated with enteral CORM-A1 (Groups III and IV). Postictal cerebral vascular function is expressed as \% of responses in the intact control (Group I). $N=5$ piglets in each group. ${ }^{\dagger} P<0.05$ compared with the intact control (Group I). ${ }^{*} P<0.05$ compared with the saline seizures group (Group II).

antioxidant therapy. We found no evidence of sex-related differences in cerebral vasodilator functions involving the endothelial, astrocytic, or vascular smooth muscle 


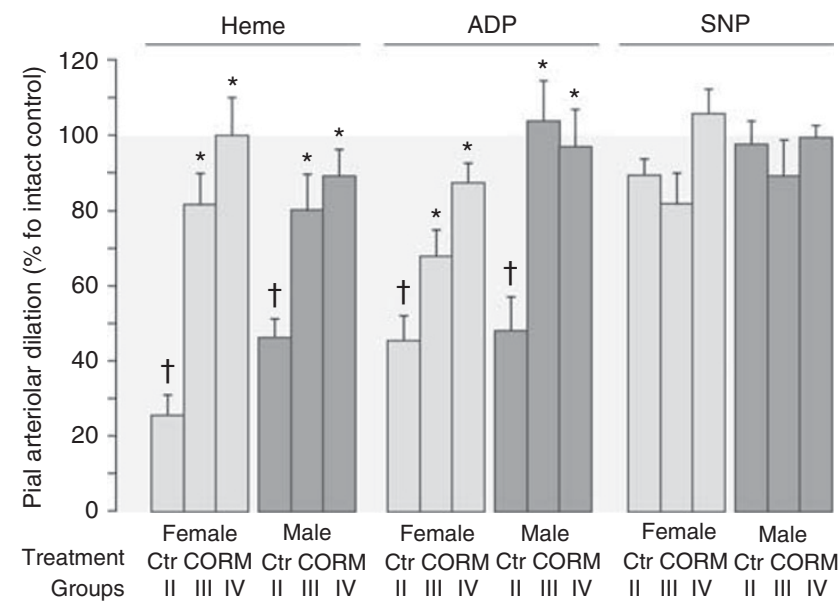

Figure 5. CORM-A1 therapy prevents postictal loss of cerebral vascular responses to heme and ADP in female and male newborn pigs (Groups III and IV). CORM-A1 (2 mg/kg) was administered $10 \mathrm{~min}$ before (Group III) or 20 min after bicuculline (Group IV). Cerebral vascular responses to topical endothelium/astrocyte-dependent dilator heme $\left(10^{-5} \mathrm{M}\right)$, astrocyte-dependent dilator $\operatorname{ADP}\left(10^{-4} \mathrm{M}\right)$ and to endothelium/ astrocyte-independent dilator sodium nitroprusside (SNP, $10^{-6} \mathrm{M}$ ) were examined $48 \mathrm{~h}$ after seizures in piglets, either untreated (Group II) or treated with enteral CORM-A1 (Groups III and IV). Postictal cerebral vascular function is expressed as \% of control responses in the intact control (Group I). $N=5$ piglets in each group. ${ }^{\dagger} P<0.05$ compared with the intact control (Group I). ${ }^{*} P<0.05$ compared with the saline seizures group (Group II).

components of the neurovascular unit in healthy and epileptic newborn pigs. In both female and male newborn pigs, epileptic seizures lead to prolonged postictal cerebral vascular dysfunction that was effectively prevented by antioxidant therapy with the CO-donor CORM-A1.

\section{Controversial Evidence of Sexual Dimorphism in Neonatal Cerebral Circulation}

The issue of sex-specific differences in neonatal cerebral circulation during physiological and pathological conditions remains highly controversial. No systematic studies have been conducted to evaluate sex-related specifics in CBF regulation in healthy neonatal brain. The majority of experimental studies were designed to uncover potential sex dimorphysm in the outcome of neonatal brain disease and to determine whether distinct approaches are needed for personalized therapy in female and male neonates. In healthy term infants, only subtle differences in regional CBF parameters in male and female subjects were detectable within 1-3 days of birth (18). Animal studies suggest that male infants have a tendency toward worse outcome of brain injury caused by inflammation, hypoxia-ischemia, stroke, and blunt trauma (16-23). However, some reports indicate that the female rat neonatal brain has high susceptibility to hypoxic-ischemic insults (36). Acute CBF impairment after fluid percussion brain injury in newborn pigs also pinpointed sex-dependent variables in outcomes and sensitivity to therapy $(16,37)$. Poor neurodevelopmental outcome of inflammatory neonatal brain disease in human male infants has been reported in several studies.
Male newborn babies exposed to maternal chorioamniotitis caused by bacterial infection showed greater CBF abnormalities that correlated with an increased risk of periventricular leukomalacia and cerebral palsy (18). Upregulated inflammatory response secondary to hypoxic-ischemic injury and a greater brain tissue loss was observed in male neonatal rats, whereas acute primary response to the injury was equivalent in male and female pups (20). However, recent large-scale clinical trials found no gender relevance to incidence and adverse neurological outcome in human infants with hypoxic-ischemic encephalopathy $(31,33,34)$. The differences between human and rodent neonatal brain may contribute to this controversy.

Apart from correlation analysis, very little is known about the mechanisms underlying purported sex-specific differences in neonatal brain injury. Although females appear to be more resilient to hypoxic-ischemic brain injury and stroke, estrogen treatment in adults and newborns did not produce noticeable neuroprotection. Sex-biased gene expression may contribute to sex-dependent differences in various physiological functions (13-15). Sex-related changes in brain mitochondria (36), nitric oxide-mediated vascular mechanisms (38), phosphorylation-mediated signaling $(16,17,37)$, and the mechanisms of cell death by apoptosis and autophagy (39) may contribute to the pathophysiology of neonatal brain disease. Considering a plethora of regulatory mechanisms that may contribute to brain injury, finding the primary key elements that are responsive to cerebrovascular dysregulation caused by various pathophysiological conditions represents a major challenge for future investigation in translationally valuable animal models.

\section{Cerebrovascular Functions in Healthy Neonates: No Sexual Dimorphism is Observed}

We performed a systematic detailed evaluation of various cerebral vasodilator functions related to endothelium- and astrocyte-dependent and -independent components of CBF regulation in healthy control female and male newborn pigs. Endothelium-dependent regulatory influences on CBF were tested by responses of pial arterioles to hypercapnia and bradykinin, whereas astrocyte-dependent influences were evaluated by responses to ADP $(5,7,8,35)$. Cerebral vascular responses that required both endothelium- and astrocytedependent influences were tested by topically applied glutamate and the AMPA receptor agonist quisqualate, and by heme, the substrate for heme oxygenase that catalyzes brain CO production $(5,8)$. The NO donor SNP was used for detecting cGMP-dependent vascular smooth muscle responses that do not require endothelial or astrocytic influences. In control healthy newborn pigs, we found no evidence of any sex-related differences in cerebral vasodilator functions involving endothelial, astrocytic, or vascular smooth muscle components of the neurovascular unit. 


\section{Epileptic Seizures Cause Sustained Dysregulation of Endothelium- and Astrocyte-Dependent Cerebrovascular Functions: No Sexual Dimorphism is Observed}

We asked a question whether sexual dimorphism contributes to long-term impairment of cerebrovascular functions and CBF dysregulation caused by epileptic seizures. During the delayed postictal period $(48 \mathrm{~h})$, cerebral vascular functions related to endothelium- and/or astrocyte-dependent CBF regulation were significantly reduced in both female and male newborn piglets (40-70\% reduction) as compared to the responses in healthy female and male controls. Cerebral vascular smooth muscle function was not adversely affected by seizures in either sex, as determined by cerebral vasodilation to SNP. These data suggest that in both female and male newborn pigs, epileptic seizures produce longlasting injurious effects on the endothelial and astrocytic components of the $\mathrm{CBF}$ regulation, whereas smooth muscle functions remain unharmed.

\section{Antioxidant Therapy with CORM-A1 Prevents Cerebral Vascular Disease Caused by Epileptic Seizures: No Sexual Dimorphism is observed}

The sex differences in responsiveness of neonates to neuroprotective therapy cannot be excluded. In some models of neonatal brain injury by hypoxia-ischemia, the efficiency of preventive treatments reveal sex differences that might be translated to introducing a sex-specific neuroprotective therapy in neonatal practice $(19-21,23,26)$. The treatment of impaired cerebral autoregulation after traumatic neonatal brain injury with vasopressor agents also reveals sex- and agedependent differences $(16,17)$.

We aimed at testing whether therapeutic approaches for preventing cerebrovascular injury caused by neonatal seizures should be applied in a sex-dependent manner. Brain oxidative stress is the key contributor to cerebrovascular dysfunction caused by seizures $(5,6,40)$. Treatment with antioxidants, including ROS scavengers Tiron and bilirubin, and NADPH oxidase inhibitor apocynin largely prevented postictal cerebrovascular dysfunction in newborn piglets of both sexes (6). In cultured cerebral vascular endothelial cells and cortical astrocytes, antioxidants reduced cell death by apoptosis caused by excitotoxic and inflammatory mediators (40). Carbon monoxide, endogenously produced in the brain, or delivered by CO-releasing pharmacological compound (CORM-A1), has antioxidant and antiapoptotic properties that provide very efficient protection against neurovascular cell death induced by oxidative stress $(6-8,40)$.

We present first experimental evidence that the therapeutic efficacy of CORM-A1 against harmful cerebrovascular effects of neonatal seizures is exhibited in a sex-independent manner. CORM-A1, administered enterally during the pre-ictal period, prevented long-term cerebral vascular dysfunction caused by seizure-induced endothelial and astrocytic injury in both female and male piglets. Importantly, efficient cerebrovascular protection in both sexes was also achieved when CORM-A1 was administered during the ictal phase. These data emphasize the high therapeutic potency of CORM-A1 against $\mathrm{CBF}$ dysregulation in both female and male newborns.

Overall, we found no sex-related differences in endothelium-, astrocyte-, and vascular smooth muscle-dependent components of $\mathrm{CBF}$ regulation in healthy newborn pigs. In both female and male piglets, epileptic seizures produced long-term cerebral vascular dysfunction related to the injury of endothelium- and astrocyte-dependent components of CBF regulation without directly affecting vascular smooth muscle. Furthermore, we found no sexual dimorphism in the effectiveness of antioxidant therapy with the CO-donor compound that prevents harmful effects of neonatal seizures on $\mathrm{CBF}$ regulation.

\section{STATEMENT OF FINANCIAL SUPPORT}

The study was supported by National Institutes of Health, Bethesda, MD RO1HL99655 (HP) and RO1HL034059 (CWL).

Disclosure: The authors declare no conflict of interest.

\section{REFERENCES}

1. Abend NS, Wusthoff CJ. Neonatal seizures and status epilepticus. J Clin Neurophysiol 2012;29:441-8.

2. Agarwal M, Fox SM. Pediatric seizures. Emerg Med Clin North Am 2013;31:733-54.

3. Clancy RR. Summary proceedings from the neurology group on neonatal seizures. Pediatrics 2006;117:S23-7.

4. Uria-Avellanal C, Marlow N, Rennie JM. Outcome following neonatal seizures. Semin Fetal Neonatal Med 2013;18:224-32.

5. Parfenova H, Carratu P, Tcheranova D, et al. Epileptic seizures cause extended postictal cerebral vascular dysfunction that is prevented by HO-1 overexpression. Am J Physiol Heart Physiol 2005;288:H2843-50.

6. Parfenova $\mathrm{H}$, Leffler CW, Basuroy S, et al. Antioxidant roles of heme oxygenase, carbon monoxide, and bilirubin in cerebral circulation during seizures. J Cereb Blood Flow Metab 2012;32:1024-34.

7. Parfenova H, Leffler CW, Tcheranova D, et al. Epileptic seizures increase circulating endothelial cells in peripheral blood as early indicators of cerebral vascular damage. Am J Physiol Heart Circ Physiol 2010;298: H1687-98.

8. Liu J, Fedinec A, Leffler CW, Parfenova H. Enteral supplements of a carbon monoxide donor CORM-A1 protect against cerebrovascular dysfunction caused by neonatal seizures. J Cereb Blood Flow Metab 2015;35:193-9.

9. Pourcyrous M, Basuroy S, Tcheranova D, et al. Brain-derived circulating endothelial cells in peripheral blood of newborn infants with seizures: a potential biomarker for cerebrovascular injury. Physiol Rep 2015;3 pii e12345.

10. Kamali AW, Cockerell OC, Butlar P. Aneurysms and epilepsy: an increasingly recognised cause. Seizure 2004;13:40-4.

11. Turjman F, Massoud TF, Sayre JW, et al. Epilepsy associated with cerebral arteriovenous malformations: a multivariate analysis of angioarchitectural characteristics. Am J Neuroradiol 1995;16:345-50.

12. Konkle AT, McCarthy MM. Developmental time course of estradiol, testosterone, and dihydrotestosterone levels in discrete regions of male and female rat brain. Endocrinology 2011;152:223-35.

13. Ober C, Loisel DA, Gilad Y. Sex-specific genetic architecture of human disease. Nat Rev Genet 2008;9:911-22.

14. Arnold AP, Burgoyne PS. Are XX and XY brain cells intrinsically different? Trends Endocrinol Metab 2004;15:6-11.

15. Ngun TC, Ghahramani N, Sánchez FJ, et al. The genetics of sex differences in brain and behavior. Front Neuroendocrinol 2011;32: $227-46$.

16. Armstead WM, Kiessling JW, Bdeir K, et al. Adrenomedullin prevents sex-dependent impairment of autoregulation during hypotension after 
piglet brain injury through inhibition of ERK MAPK upregulation. J Neurotrauma 2010;27:391-402.

17. Armstead WM, Riley J, Vavilala MS. Preferential protection of cerebral autoregulation and reduction of hippocampal necrosis with norepinephrine after traumatic brain injury in female piglets. Pediatr Crit Care Med 2016;17:e130-7.

18. Koch FR, Wagner CL, Jenkins DD, et al. Sex differences in cerebral blood flow following chorioamnionitis in healthy term infants. J Perinatol 2014;34:197-202.

19. Mayoral SR, Omar G, Penn AA. Sex differences in a hypoxia model of preterm brain damage. Pediatr Res 2009;66:248-53.

20. Mirza MA, Ritzel R, Xu Y, McCullough LD, Liu F. Sexually dimorphic outcomes and inflammatory responses in hypoxic-ischemic encephalopathy. J Neuroinflammation 2015;12:32.

21. Smith AL, Alexander M, Rosenkrantz TS, et al. Sex differences in behavioral outcome following neonatal hypoxia ischemia: insights from a clinical meta-analysis and a rodent model of induced hypoxic ischemic brain injury. Exp Neurol 2014;254:54-67.

22. Tontisirin N, Muangman SL, Suz P, et al. Early childhood gender differences in anterior and posterior cerebral blood flow velocity and autoregulation. Pediatrics 2007;119:e610-5.

23. Waddell J, Hanscom M, Shalon Edwards N, et al. Sex differences in cell genesis, hippocampal volume and behavioral outcomes in a rat model of neonatal HI. Exp Neurol 2016;275:285-95.

24. Vohr BR, Wright LL, Poole WK, McDonald SA. Neurodevelopmental outcomes of extremely low birth weight infants b32 weeks' gestation between 1993 and 1998. Pediatrics 2005;116:635-43.

25. Jacobs SE, Berg M, Hunt R, et al. Cooling for newborns with hypoxic ischaemic encephalopathy. Cochrane Database Syst Rev 2013;1: CD003311.

26. Cohen SS, Stonestreet BS. Sex differences in behavioral outcome following neonatal hypoxia ischemia: insights from a clinical meta-analysis and a rodent model of induced hypoxic ischemic injury. Exp Neurol 2014;256:70-3.

27. Akman O, Moshé SL, Galanopoulou AS. Sex-specific consequences of early life seizures. Neurobiol Dis 2014;72:153-66.

28. Atif F, Yousuf S, Stein DG. Progesterone in the treatment of neonatal arterial ischemic stroke and acute seizures: role of BDNF/TrkB signaling. Neuropharmacology 2016;107:317-28.
29. Kang SK, Markowitz GJ, Kim ST, et al. Age- and sex-dependent susceptibility to phenobarbital-resistant neonatal seizures: role of chloride co-transporters. Front Cell Neurosci 2015;9:173.

30. VelíškováJ,DesantisKA. Sex and hormonal influences on epilepsy. Horm Behav 2013;63:267-77.

31. Guillet R, Edwards AD, Thoresen M, et al. Seven- to eight-year follow-up of the CoolCap trial of head cooling for neonatal encephalopathy. Pediatr Res 2012;71:205-9.

32. Saliba RM, Annegers FJ, Waller DK, et al. Risk factors for neonatal seizures: a population-based study, Harris County, Texas, 1992-1994. Am J Epidemiol 2001;154:14-20.

33. Shankaran S, Pappas A, McDonald SA, et al. Childhood outcomes after hypothermia for neonatal encephalopathy. N Engl J Med 2012;36: 2085-92.

34. Shellhaas RA, Chang $\mathrm{T}$, Tsuchida $\mathrm{T}$, et al. The American Clinical Neurophysiology Society's Guideline on Continuous Electroencephalography Monitoring in Neonates. J Clin Neurophysiol 2011;28: 611-7.

35. Harsono M, Pourcyrous M, Jolly EJ, et al. Selective head cooling during neonatal seizures prevents postictal cerebral vascular dysfunction without reducing epileptiform activity. Am J Physiol Heart Circ Physiol 2016;311: H1202-13.

36. Weis SN, Pettenuzzo LF, Krolow R, et al. Neonatal hypoxia-ischemia induces sex-related changes in rat brain mitochondria. Mitochondrion 2012;12:271-9.

37. Zhu C, Sun Y, Gao J, et al. Inhaled nitric oxide protects males but not females from neonatal mouse hypoxia-ischemia brain injury. Transl Stroke Res 2013;4:201-7.

38. Kosty J, Riley J, Liang J, Armstead WM. Influence of Sex and ERK MAPK on the Pressure Reactivity Index in newborn piglets after fluid percussion injury. Transl Stroke Res 2012;3:460-5.

39. Weis SN, Toniazzo AP, Ander BP, et al. Autophagy in the brain of neonates following hypoxia-ischemia shows sex- and region-specific effects. Neuroscience 2014;256:201-9.

40. Basuroy S, Leffler CW, Parfenova H. CORM-A1 prevents blood-brain barrier dysfunction caused by ionotropic glutamate receptor-mediated endothelial oxidative stress and apoptosis. Am J Physiol Cell Physiol 2013;304:C1105-15. 